\title{
High peak current operation of $x$-ray free-electron laser multiple beam lines by suppressing coherent synchrotron radiation effects
}

\author{
Toru Hara, ${ }^{1, *}$ Chikara Kondo, ${ }^{2}$ Takahiro Inagaki, ${ }^{1}$ Kazuaki Togawa, ${ }^{1}$ Kenji Fukami, ${ }^{2}$ \\ Shingo Nakazawa, ${ }^{3}$ Taichi Hasegawa, ${ }^{3}$ Osamu Morimoto, ${ }^{3}$ Masamichi Yoshioka, ${ }^{3}$ \\ Hirokazu Maesaka, ${ }_{1}$ Yuji Otake, ${ }^{1}$ and Hitoshi Tanaka ${ }^{1}$ \\ ${ }^{1}$ RIKEN SPring-8 Center, Kouto 1-1-1, Sayo-cho, Sayo-gun, Hyogo 679-5148, Japan \\ ${ }^{2} J a p a n$ Synchrotron Radiation Research Institute, \\ Kouto 1-1-1, Sayo-cho, Sayo-gun, Hyogo 679-5198, Japan \\ ${ }^{3}$ SPring-8 Service Co., Ltd., Kouto 1-20-5, Shingu-cho, Tatsuno, Hyogo 679-5165, Japan
}

(Received 16 February 2018; published 2 April 2018)

\begin{abstract}
The parallel operation of multiple beam lines is an important means to expand the opportunity of user experiments at $\mathrm{x}$-ray free-electron laser (XFEL) facilities. At SPring-8 Angstrom free-electron laser (SACLA), the multi-beam-line operation had been tested using two beam lines, but transverse coherent synchrotron radiation (CSR) effects at a dogleg beam transport severely limited the laser performance. To suppress the CSR effects, a new beam optics based on two double bend achromat (DBA) structures was introduced for the dogleg. After the replacement of the beam optics, high peak current bunches of more than $10 \mathrm{kA}$ are now stably transported through the dogleg and the laser pulse output is increased by a factor of 2-3. In the multi-beam-line operation of SACLA, the electron beam parameters, such as the beam energy and peak current, can be adjusted independently for each beam line. Thus the laser output can be optimized and wide spectral tunability is ensured for all beam lines.
\end{abstract}

DOI: 10.1103/PhysRevAccelBeams.21.040701

\section{INTRODUCTION}

XFELs have distinctive characteristics such as high peak intensity, short pulse duration and coherence [1-7]. They have been used in diverse scientific fields including biology, physics, chemistry, and material science [8-11]. Since XFELs require a high brightness electron beam, a linear accelerator is used as an electron beam driver. Consequently the number of operational beam lines is limited to one, which has been a drawback of XFEL facilities in contrast to synchrotron radiation sources having many beam lines operated in parallel around a storage ring. In order to mitigate this drawback, multi-beam-line operation has been tested at SACLA by distributing the electron beam to multiple beam lines from bunch to bunch [12].

Figure 1 shows a schematic of the SACLA facility [2]. The undulator hall can accommodate up to five beam lines and two of them (BL2 and BL3) are currently in operation as XFEL beam lines. BL1 is a dedicated soft X-ray FEL beam line driven by the former SCSS test accelerator and independently running from the XFEL beam lines [13-15].

\footnotetext{
*toru@spring8.or.jp
}

Published by the American Physical Society under the terms of the Creative Commons Attribution 4.0 International license. Further distribution of this work must maintain attribution to the author(s) and the published article's title, journal citation, and DOI.
For the multi-beam-line operation, the electron bunches from the SACLA linear accelerator are alternately sent to the two XFEL beam lines, BL2 and BL3 [12]. For BL3, the electron beam travels straight from the end of the linear accelerator, and the electron beam is deflected by $3^{\circ}$ at a switchyard for BL2. There is another beam transport line called XSBT (XFEL to Synchrotron Beam Transport) used for the beam injection to the SPring-8 storage ring, for which the electron beam is deflected by $-3^{\circ}$, in opposite direction of BL2, at the switchyard.

The first multi-beam-line operation of SACLA was tested using a kicker and a DC septum magnet [12]. The deflection angle of the kicker was made as small as possible to ease stability requirements for its pulsed power supply, so the electron beam was deflected by $0.5^{\circ}$ at the kicker and $2.5^{\circ}$ at the DC septum magnet. Consequently the electron beam optics of the $3^{\circ}$ dogleg beam transport became asymmetric and the cancellation of transverse CSR effects was neglected. Although parallel lasing had been successfully achieved at the two XFEL beam lines, stable lasing was only obtained with reduced peak currents lower than $3 \mathrm{kA}$ due to the CSR effects of the dogleg with this old beam optics.

For the single beam line operation of SACLA, the electron bunches are longitudinally compressed to $10-15$ fs (FWHM) and the peak current reaches more than $10 \mathrm{kA}$. When these high peak current bunches pass through the dogleg, the electron beam orbit instability and projected emittance 


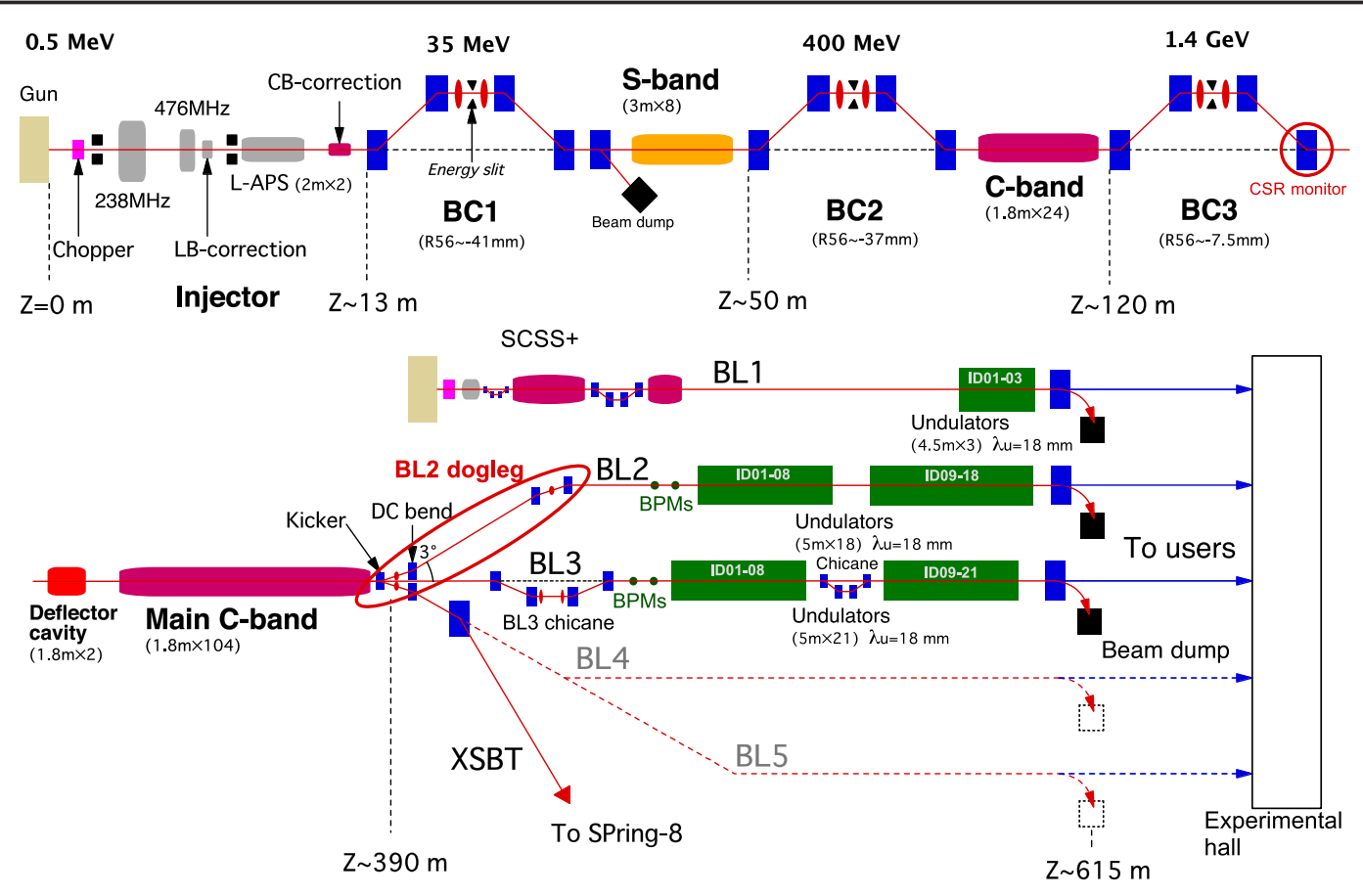

FIG. 1. Schematic layout of SACLA.

growth in the deflecting plane occur [16,17]. In order to avoid the degradation of the electron beam quality, a new beam optics is introduced to the BL2 dogleg beam transport.

\section{CANCELLATION OF CSR EFFECTS}

When an electron bunch is deflected in a dipole magnet, synchrotron radiation is emitted and its spectral components comparable to the bunch length become coherent [18]. Then the CSR fields emitted from the tail electrons interact with the head electrons inside the same electron bunch during the travel through the dipole magnet and its downstream [16]. The CSR fields have both transverse and longitudinal components with respect to the direction of movement of the electron. The transverse component directly adds a transverse kick to the electron in the plane of deflection, while the longitudinal component changes the electron energy. Once the electron energy is changed inside the dipole magnet, the electron orbit deviates from a reference orbit and namely CSR dispersion, which differs from ordinary momentum dispersion, arises [19]. In case of the dogleg beam transport of SACLA, the effect due to the longitudinal component is dominant, which is confirmed by the fact that 1-D and 3-D simulations give no significant difference $[20,21]$. In order to avoid these transverse effects of CSR, it is necessary to cancel out the transverse kicks and the CSR dispersion at the end of the dogleg beam transport.

The use of two sets of DBA structures is the simplest solution to cancel out the CSR effects of the dogleg [22,23]. By making the betatron phase advance between adjacent dipoles an odd multiple of $\pi$ in the deflecting plane, the sum of the CSR dispersion becomes zero at the end of the dogleg. Since the betatron phase advance between the two dipoles of DBA is naturally $\pi$, only the phase advance between the two DBA structures is adjusted to $\pi$. In this case, the transverse CSR kicks are also cancelled at the end of the dogleg.

For the cancellation of the CSR effects, it is necessary to keep the CSR fields the same for all dipole magnets. Therefore at SACLA, all dipole magnets are made identical and the longitudinal current profile of the electron bunch is maintained the same. The electron bunch length of SACLA used for the daily operation is about 10 fs (FWHM), corresponding to $3 \mu \mathrm{m}$, with an energy chirp of the order of $1 \times 10^{-3}$. Although $R_{56}$ of the DBA based dogleg is as small as $450 \mu \mathrm{m}$, the change of the current profile is not negligible. In order to minimize the change of the longitudinal current profile, $R_{56}$ is set to zero for each DBA structure by making the electron beam orbit off-centered at the quadrupole magnets of the DBA structures.

\section{NEW BEAM SWITCHYARD AND DOGLEG BEAM TRANSPORT}

Figure 2 shows the magnet configuration and the beam optics functions of the new BL2 dogleg beam transport. The electron beam from the linear accelerator is first deflected by two $1.5^{\circ}$ dipoles of the DBA structure, and the beam is deflected back at the end of the dogleg to make the beam orbit parallel to the accelerator axis. The beta functions are designed to be symmetric with respect to the center of the dogleg and the horizontal beta function takes its minimum at the dipoles. 


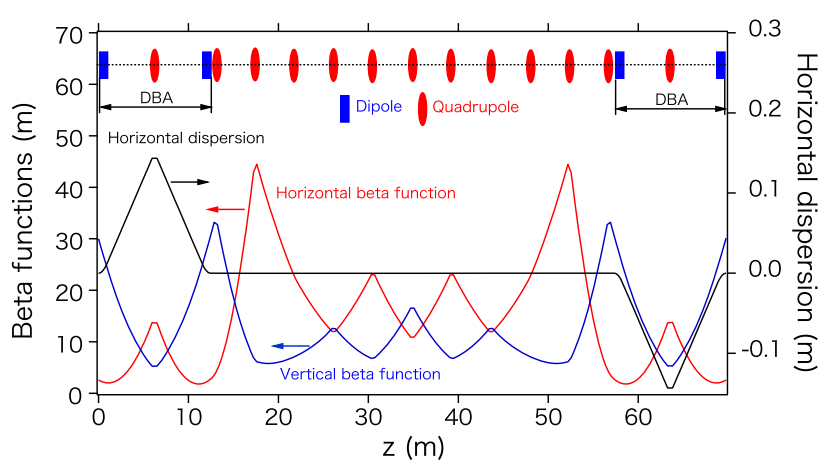

FIG. 2. Beam optics functions and magnet configuration of the BL2 dogleg beam transport. The red and blue lines show horizontal and vertical beta functions and the black line is a horizontal dispersion function.

The first dipole of the DBA structures is a kicker magnet, which works as a beam switchyard and deflects the electron beam in three directions. A pulsed power supply of the new kicker employs SiC MOSFETs as switching elements and generates trapezoidal current waveforms at $60 \mathrm{~Hz}$, which is the maximum bunch repetition of the SACLA accelerator [24]. The amplitude and polarity of the trapezoidal waveform can be changed from pulse to pulse according to the beam energy and the direction of beam deflection.

Since the stability of the electron beam orbit at undulators is crucially important for XFELs, the stability of the kicker magnetic fields were checked before installation in the accelerator tunnel. The magnetic fields at the flat top of the trapezoidal waveform, where the electron beam is deflected, were measured using a gated NMR detector [25]. As in Fig. 3, the measured pulse-to-pulse stability is about $10 \mathrm{ppm}$ (in full width). Since the deflection angle of the kicker is $1.5^{\circ}, 10 \mathrm{ppm}$ field variation corresponds to $0.26 \mu \mathrm{rad}$ and it is acceptable compared with the intrinsic orbit stability of the SACLA accelerator, which is typically $1 \mu \mathrm{rad}$ (peak to peak). Although there is a slow drift in Fig. 3, the resulting electron beam orbit change can be corrected using a beam orbit feedback system.

The cancellation of the CSR effects is confirmed by ELEGANT simulations [20]. Figure 4 shows the horizontal

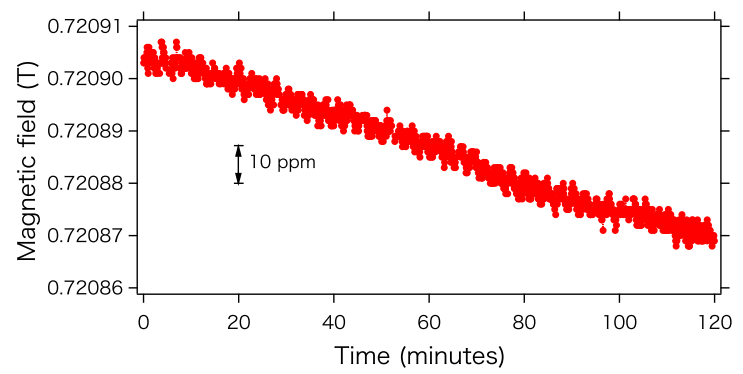

FIG. 3. Stability of the kicker magnetic fields. The kicker runs at $60 \mathrm{~Hz}$ and the sampling interval of the pulsed magnetic fields is 3 seconds.
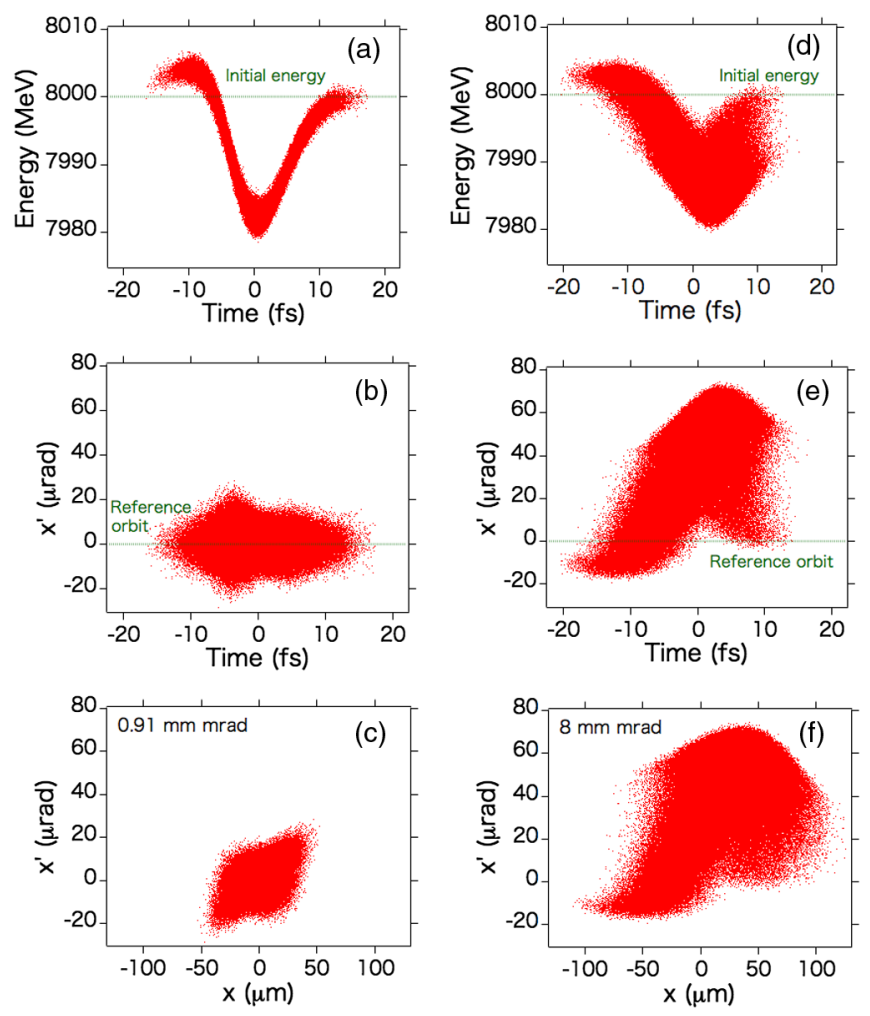

FIG. 4. Phase space distributions of the electron bunch after the BL2 dogleg. $(\mathrm{a}-\mathrm{c})$ and $(\mathrm{d}-\mathrm{f})$ are calculated for the new beam optics and for the old beam optics, respectively. The initial conditions of the electron bunch are $8 \mathrm{GeV}, 10 \mathrm{kA}, 10 \mathrm{fs}$ (FWHM) and $0.8 \mathrm{~mm} \mathrm{mrad}$.

electron distributions in the phase spaces after the dogleg. Figures $4(\mathrm{a}-\mathrm{c})$ and $4(\mathrm{~d}-\mathrm{f})$ are calculated for the new beam optics with four identical dipoles and for the old beam optics with three non-identical dipoles, respectively. The initial conditions of the electron bunch assumed in Fig. 4 are a Gaussian distribution with a $10 \mathrm{kA}$ peak current, a 10 fs bunch length (FWHM), and normalized projected emittance of $0.8 \mathrm{~mm}$ mrad.

With the old beam optics, the electron bunch is locally kinked due to the energy loss along the bunch by the CSR fields [12]. As a result, the projected emittance increases by an order of magnitude. On the other hand, the emittance growth is suppressed within $10 \%$ for the new beam optics. Although $R_{56}$ is adjusted to zero for the entire dogleg transport, the bunch length varies locally inside the DBA structure, which prevents the perfect cancellation of the CSR effects.

As seen in Figs. 4(b) and 4(e), the horizontal center of the electron bunch is the same as a reference orbit with the new beam optics, while it deviates largely with the old optics leading to the horizontal orbit instability caused by peak current variation.

In Fig. 5, the normalized horizontal emittance of the new beam optics is calculated as a function of the horizontal phase advance between the two DBA structures. The phase 


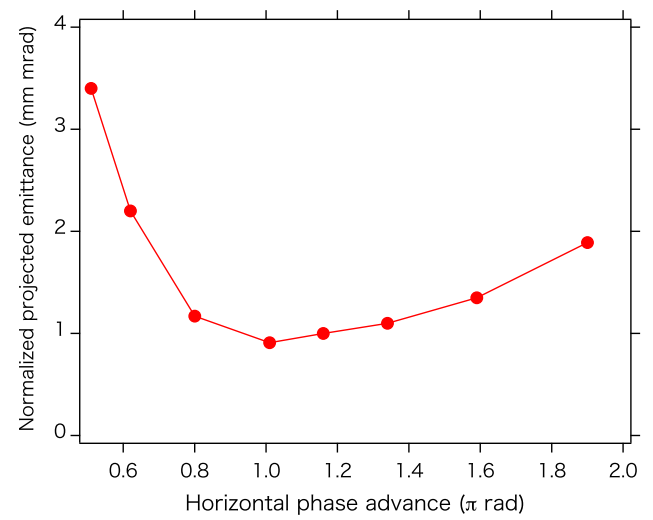

FIG. 5. Normalized horizontal projected emittance after the BL2 dogleg calculated as a function of the horizontal phase advance between the two DBA structures. Initial conditions of the electron beam are the same as Fig. 4.

advance is varied by uniformly scaling the 11 quadrupole magnets between the two DBA structures of the BL2 dogleg. As expected, the CSR effects are cancelled for a $\pi$ phase advance giving the minimum emittance growth.

\section{MULTI-BEAM-LINE OPERATION}

The suppression of the CSR effects is experimentally confirmed by measuring the electron beam orbit stability after the BL2 dogleg. The beam position and angle are measured using two beam position monitors (BPMs) in front of the BL2 undulators (Fig. 1). Figure 6 is the measured stability of the electron beam orbit in the phase space. The electron beam energy is $7.9 \mathrm{GeV}$ and the peak current is around $10 \mathrm{kA}$. The horizontal beam orbit scatters over an area of $16 \mathrm{pm}$ rad with the old beam optics, while
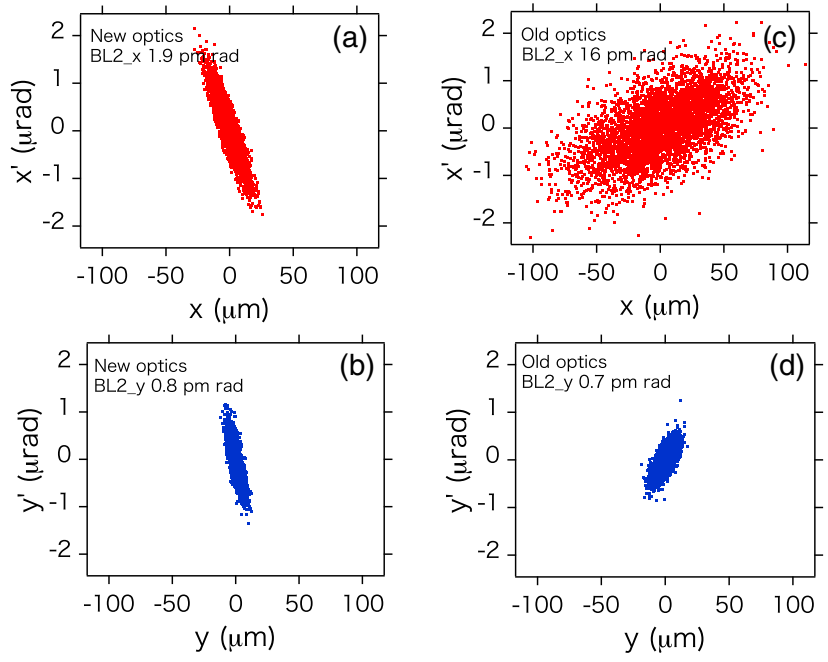

FIG. 6. Stability of the electron beam orbit after the BL2 dogleg measured with $(a, b)$ the new beam optics and $(c, d)$ the old beam optics. The beam energy and peak current of the electron bunches are $7.9 \mathrm{GeV}$ and about $10 \mathrm{kA}$.

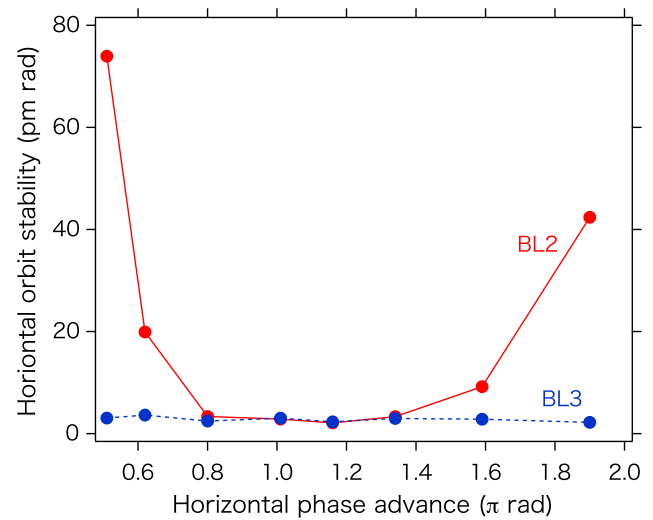

FIG. 7. Stability of the horizontal electron beam orbit as a function of the horizontal phase advance between the two DBA structures of the BL2 dogleg. The red solid circles show the stability of the electron beam orbit in the horizontal phase space measured in front of the BL2 undulators. The horizontal orbit stability of BL3, which is not affected by the CSR effects, is also shown by the blue solid circles for reference.

the scattered area decreases by an order of magnitude and the transverse CSR effects are suppressed with the new beam optics. Compared with the vertical orbit stability, which is not affected by the CSR effects, the horizontal orbit stability is worse by several times, but it is an acceptable level for XFEL operation.

Figure 7 is the measured stability of the electron beam orbit in the horizontal phase space as a function of the horizontal phase advance between the two DBA structures of the BL2 dogleg. The strength of the 11 quadrupole magnets are changed in the same way as in Fig. 5. When the phase advance deviates from $\pi$, the CSR effects of the two DBA structures do not cancel to each other and the electron beam orbit becomes unstable. The blue solid circles in Fig. 7 are the horizontal orbit stability of BL3 simultaneously measured in front of the BL3 undulators, which is not affected by the CSR effects and shows natural orbit stability of the accelerator.

Regarding the XFEL output, the decrease of the pulse energy is less than $30 \%$ for the phase advance between $0.9 \pi$ and $1.1 \pi$. A comparable pulse energy drop is observed by changing $R_{56}$ of the dogleg between $-100 \mu \mathrm{m}$ and $+100 \mu \mathrm{m}$.

Owing to the cancellation of the CSR effects, BL2 and BL3 are now operated in parallel using the high peak current bunches of more than $10 \mathrm{kA}$. Figure 8 shows the laser pulse energies of the two beam lines. The electron beam energy is $7.8 \mathrm{GeV}$ and the K-parameters of the undulators are 2.1. In Fig. 8, $60 \mathrm{~Hz}$ electron bunches are alternatively deflected to BL2 and BL3, so the laser pulse repetition at each beam line is $30 \mathrm{~Hz}$.

When operating the two beam lines in parallel, the optimum conditions of the electron bunch compression with respect to the laser output might slightly differ for the 

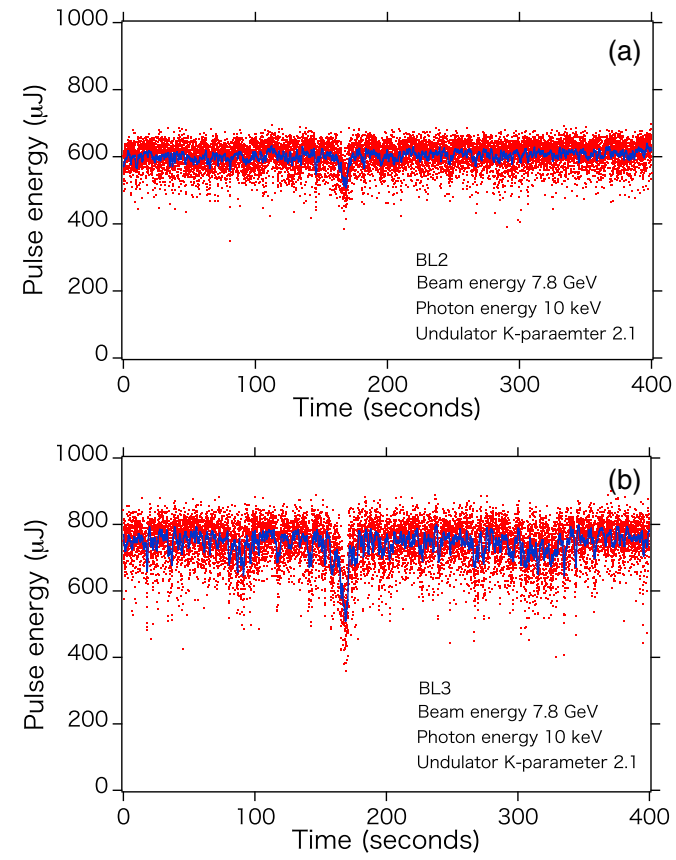

FIG. 8. XFEL pulse energies obtained in the multi-beam-line operation, (a) BL2 and (b) BL3. The photon energy is $10 \mathrm{keV}$. The red dots represent single-shot results and the blue lines show averaged values over one second. The electron beam energy is $7.8 \mathrm{GeV}$ and the repetition of the electron beam is $60 \mathrm{~Hz}$. K-parameters of the undulators are set to 2.1 for both BL2 and BL3.

two beam lines. This is mainly due to the different $R_{56}$ of BL2 and BL3. As mentioned previously, $R_{56}$ of the BL2 dogleg is adjusted to zero for the cancellation of the CSR effects, while there is a chicane, whose $R_{56}$ is about $-750 \mu \mathrm{m}$, between the switchyard and the BL3 undulators to measure the final electron beam energy and remove dark currents from C-band accelerating structures (Fig. 1). Therefore, the longitudinal distribution of the electron bunch of BL3 is slightly affected by the chicane. When maximizing the XFEL pulse energies, the optimum conditions of the electron bunch compression become different for BL2 and BL3.

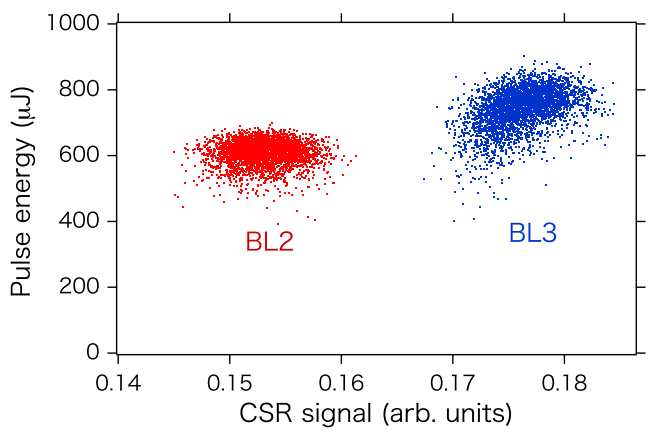

FIG. 9. XFEL pulse energies of BL2 and BL3 measured as a function of the CSR signal at BC3. The red and blue dots represent the pulses of BL2 and BL3, respectively.

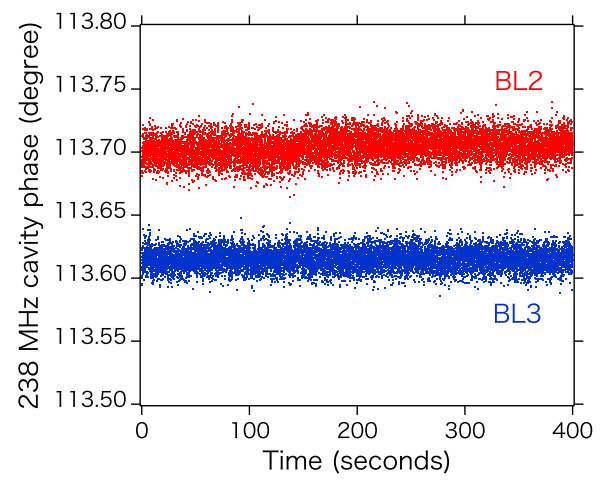

FIG. 10. RF phase of a $238 \mathrm{MHz}$ cavity. The phases of $60 \mathrm{~Hz}$ RF pulses are alternately changed between the optimum values of BL2 (red dots) and BL3 (blue dots).

In Fig. 9, the laser pulse energies of BL2 and BL3 are plotted with respect to the CSR intensity, which relates with the longitudinal distribution of the electron bunch, measured at the final bunch compressor (BC3 in Fig. 1) [26]. From Fig. 9, the optimum conditions of the bunch compression are apparently different between the two beam lines. At SACLA, the bunch compression parameters, namely rf phases, can be changed from bunch to bunch and optimized independently for the two beam lines to maximize the laser pulse energies. Figure 10 shows the rf phase
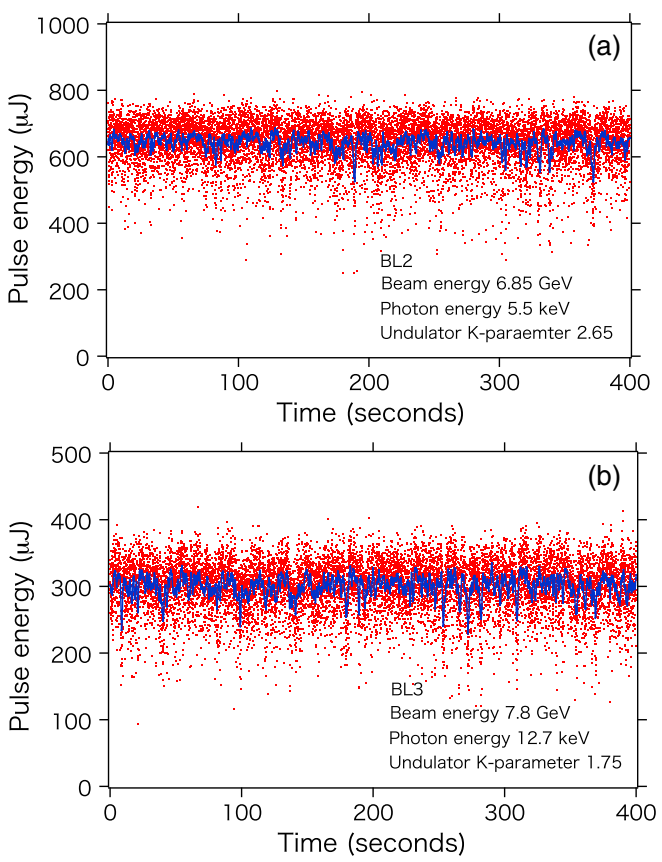

FIG. 11. XFEL pulse energies obtained in the multi-beam-line operation, (a) BL2 and (b) BL3. The photon energies are $5.5 \mathrm{keV}$ at BL2 and $12.7 \mathrm{keV}$ at BL3. The red dots represent single-shot results and the blue lines show averaged values over one second. The beam repetition is $60 \mathrm{~Hz}$. The electron beam energy and K-parameter are $6.85 \mathrm{GeV}$ and 2.65 for BL2 and $7.8 \mathrm{GeV}$ and 1.75 for BL3. 
of a $238 \mathrm{MHz}$ cavity of the injector section. The phases of $60 \mathrm{~Hz}$ rf pulses are alternately changed between two values to operate the two beam lines at their optimum phases.

Spectral tunability is one of the important features of FELs. Since SACLA employs in-vacuum variable gap undulators with an $18 \mathrm{~mm}$ magnetic period, the photon energies of the two beam lines can be independently tuned through the undulator gap or K-parameter. In addition to the K-parameters, the electron beam energies can be also changed between the beam lines to achieve wider spectral tunability at SACLA [27].

In Fig. 11, the electron bunches are alternately accelerated to 6.85 and $7.8 \mathrm{GeV}$ by running fourteen C-band accelerating structures at $30 \mathrm{~Hz}$, which is half the repetition of the electron bunches. Then the kicker deflects the lower energy bunches to BL2 and the higher energy bunches to BL3. Thus a wide spectral tuning range more than a factor of two between the beam lines can be covered in the multibeam-line operation.

\section{SUMMARY}

The new beam optics of the BL2 dogleg beam transport based on two DBA structures successfully suppresses the transverse CSR effects, and the high peak current electron bunches are stably transported to the BL2 undulators. As a result, the laser pulse energy of BL2 is increased by a factor of 2-3 compared to that with the old beam optics.

In the multi-beam-line operation of SACLA, the electron beam energy and the parameters of the bunch compression can be changed from bunch to bunch and optimized independently for the two beamlines. Thus the maximum laser output can be obtained simultaneously at both beam lines with maintaining wide spectral tunability.

After the modification of the BL2 dogleg, full laser performance using the high peak current bunches is now available in the multi-beam-line operation. Together with the BL1 soft x-ray FEL, the parallel operation of the three beam lines considerably improves the usability of the facility and substantially expands the opportunity of XFEL user experiments.

\section{ACKNOWLEDGMENTS}

The authors wish to acknowledge the support of the SACLA team, for their help with the accelerator operation and data acquisition.

[1] P. Emma et al., First lasing and operation of an ångstromwavelength free-electron laser, Nat. Photonics 4, 641 (2010).

[2] T. Ishikawa et al., A compact X-ray free-electron laser emitting in the sub-ångström region, Nat. Photonics 6, 540 (2012).
[3] H.-S. Kang et al., Hard X-ray free-electron laser with femtosecond-scale timing jitter, Nat. Photonics 11, 708 (2017).

[4] H. Weise and W. Decking, in Proceedings of FEL2017, Santa Fe, August 2017 (to be published).

[5] C. J. Milne et al., SwissFEL: The Swiss X-ray Free Electron Laser, Appl. Sci. 7, 720 (2017).

[6] W. Ackermann et al., Operation of a free-electron laser from the extreme ultraviolet to the water window, Nat. Photonics 1, 336 (2007).

[7] E. Allaria et al., Two-stage seeded soft-X-ray free-electron laser, Nat. Photonics 7, 913 (2013).

[8] C. Bostedt, S. Boutet, D. M. Fritz, Z. Huang, H. J. Lee, H. T. Lemke, A. Robert, W. F. Schlotter, J. J. Turner, and G. J. Williams, Linac Coherent Light Source: The first five years, Rev. Mod. Phys. 88, 015007 (2016).

[9] P. Abbamonte et al., SLAC National Accelerator Laboratory Report No. SLAC-R-1053, 2015.

[10] M. Yabashi, H. Tanaka, and T. Ishikawa, Overview of the SACLA facility, J. Synchrotron Radiat. 22, 477 (2015).

[11] M. Yabashi, H. Tanaka, K. Tono, and T. Ishikawa, Status of the SACLA facility, Appl. Sci. 7, 604 (2017).

[12] T. Hara et al., Pulse-by-pulse multi-beam-line operation for X-ray free-electron lasers, Phys. Rev. Accel. Beams 19, 020703 (2016).

[13] T. Shintake et al., A compact free-electron laser for generating coherent radiation in the extreme ultraviolet region, Nat. Photonics 2, 555 (2008).

[14] S. Owada et al., A soft X-ray free-electron laser beamline at SACLA: the light source, photon beamline and experimental station, J. Synchrotron Radiat. 25, 282 (2018).

[15] K. Togawa et al., in Proceedings of IPAC2017, Copenhagen, May 2017 (2017), p. 1209.

[16] E. L. Saldin, E. A. Schneidmiller, and M. V. Yurkov, On the coherent radiation of an electron bunch moving in an arc of a circle, Nucl. Instrum. Methods Phys. Res., Sect. A 398, 373 (1997).

[17] B. E. Carlsten and T. O. Raubenheimer, Emittance growth of bunched beams in bends, Phys. Rev. E 51, 1453 (1995).

[18] R. Talman, Novel Relativistic Effect Important in Accelerators, Phys. Rev. Lett. 56, 1429 (1986).

[19] Y. Jiao, X. Cui, X. Huang, and G. Xu, Generic conditions for suppressing the coherent synchrotron radiation induced emittance growth in a two-dipole achromat, Phys. Rev. ST Accel. Beams 17, 060701 (2014).

[20] M. Borland, Simple method for particle tracking with coherent synchrotron radiation, Phys. Rev. ST Accel. Beams 4, 070701 (2001).

[21] M. Dohlus and T. Limberg, in Proceedings of FEL2004, Trieste, August 2004 (2004), p. 18.

[22] D. Douglas, Thomas Jefferson National Accelerator Facility Report No. JLAB-TN-98-012, 1998.

[23] S. Di Mitri, M. Cornacchia, and S. Spampinati, Cancellation of Coherent Synchrotron Radiation Kicks with Optics Balance, Phys. Rev. Lett. 110, 014801 (2013).

[24] C. Kondo et al., in Proceedings of IPAC2017, Copenhagen, May 2017 (2017), p. 3404. 
[25] T. Hara et al., in Proceedings of FEL2014, Bazel, August 2014 (2014), p. 71.

[26] Y. Otake, H. Maesaka, S. Matsubara, S. Inoue, K. Yanagida, H. Ego, C. Kondo, T. Sakurai, T. Matsumoto, and $\mathrm{H}$. Tomizawa, Beam monitor system for an $\mathrm{X}$-ray free electron laser and compact laser, Phys. Rev. ST Accel. Beams 16, 042802 (2013).

[27] T. Hara et al., Time-interleaved multienergy acceleration for an x-ray free-electron laser facility, Phys. Rev. ST Accel. Beams 16, 080701 (2013). 